\title{
Wolbachia lipoproteins: abundance, localisation and serology of Wolbachia peptidoglycan associated lipoprotein and the Type IV Secretion System component, VirB6 from Brugia malayi and Aedes albopictus
}

\author{
Denis Voronin, Ana F Guimarães, Gemma R Molyneux, Kelly L Johnston, Louise Ford and Mark J Taylor
}

\begin{abstract}
Background: Lipoproteins are the major agonists of Wolbachia-dependent inflammatory pathogenesis in filariasis and a validated target for drug discovery. Here we characterise the abundance, localisation and serology of the Wolbachia lipoproteins: Wolbachia peptidoglycan associated lipoprotein and the Type IV Secretion System component, VirB6.

Methods: We used proteomics to confirm lipoprotein presence and relative abundance; fractionation, immunoblotting and confocal and electron immuno-microscopy for localisation and ELISA for serological analysis.

Results: Proteomic analysis of Brugia malayi adult female protein extracts confirmed the presence of two lipoproteins, previously predicted through bioinformatics: Wolbachia peptidoglycan associated lipoprotein (wBmPAL) and the Type IV Secretion System component, VirB6 (wBmVirB6). wBmPAL was among the most abundant Wolbachia proteins present in an extract of adult female worms with wBmVirB6 only detected at a much lower abundance. This differential abundance was reflected in the immunogold-labelling, which showed WBmPAL localised at numerous sites within the bacterial membranes, whereas wBmVirB6 was present as a single cluster on each bacterial cell and also located within the bacterial membranes. Immunoblotting of fractionated extracts confirmed the localisation of WBMPAL to membranes and its absence from cytosolic fractions of C6/36 mosquito cells infected with wAlbB. In whole worm mounts, antibody labelling of both lipoproteins were associated with Wolbachia. Serological analysis showed that both proteins were immunogenic and raised antibody responses in the majority of individuals infected with Wuchereria bancrofti.
\end{abstract}

Conclusions: Two Wolbachia lipoproteins, WBmPAL and WBmVirB6, are present in extracts of Brugia malayi with WBmPAL among the most abundant of Wolbachia proteins. Both lipoproteins localised to bacterial membranes with wBmVirB6 present as a single cluster suggesting a single Type IV Secretory System on each Wolbachia cell.

Keywords: Wolbachia, Lipoprotein, Peptidoglycan associated lipoprotein, Type IV Secretion System VirB6, Brugia malayi, Aedes albopictus

\footnotetext{
* Correspondence: Mark.Taylor@lstmed.ac.uk

Department of Parasitology, Liverpool School of Tropical Medicine,

Pembroke Place, Liverpool L3 5QA, UK
} 


\section{Background}

Wolbachia are one of the most abundant intracellular symbiotic bacteria in nature. They are most common in terrestrial arthropods, with up to $40 \%$ of species infected, and are also found in a sub-group of filarial nematodes, where they have evolved a mutualistic association $[1,2]$. In filarial nematodes Wolbachia are essential for normal larval growth and development, embryogenesis and the survival of adult worms. Loss of Wolbachia induces extensive apoptosis of germline and somatic cells, presumably due to the lack of provision of an essential nutrient or metabolite required to prevent apoptosis of these cells and tissues during periods of high metabolic demand [3]. This mutualistic association has been exploited as a target for antibiotic therapy, which can cure patients infected with Wuchereria bancrofti and Onchocerca volvulus, providing an alternative treatment and control strategy $[1,4,5]$.

In addition to their essential role in the biology of filarial nematodes, Wolbachia are a major driver of the inflammatory pathogenesis of filarial disease [6]. Previous studies have determined that the pro-inflammatory capacity of Brugia malayi and Onchocerca volvulus is dependent on the presence of Wolbachia and the molecular ligands responsible have been characterised as lipoproteins [7]. The induction of innate and adaptive inflammatory responses is dependent on recognition by Toll-like receptors 2 and 6 (TLR2/6) [7,8], pattern recognition receptors for di-acylated bacterial lipoproteins. Analysis of the Wolbachia B. malayi (wBm) genome revealed the absence of the gene for the apolipoprotein $\mathrm{N}$ acyltransferase (Lnt), which converts di- to tri- acylated bacterial lipoprotein in gram-negative bacteria, suggesting lipoproteins in Wolbachia are di-acylated accounting for their recognition by TLR2/6 [7].

Bioinformatic analysis of $w \mathrm{Bm}$ genome using three distinct databases consistently identified only two lipoproteins [7]: 1) Wolbachia peptidoglycan-associated lipoprotein (wBmPAL) and 2) Wolbachia VirB6 (wBmVirB6), which is a core component of the bacterial Type IV secretion system (TIVSS) [9]. Furthermore, a chemically synthesised Wolbachia PAL di-acylated lipopeptide replicated the inflammatory activity of whole Wolbachia and soluble parasite extracts [7]. Here, we have further characterised these two Wolbachia lipoproteins to determine their relative abundance, structural localisation in the filarial nematode Brugia malayi (naturally infected with $w \mathrm{Bm}$ ) and Ae. albopictus cell line (C6/36 infected with wAlbB) and serology in people infected with W. bancrofti.

\section{Methods}

\section{Parasite material, mosquito cell line culture and patient} serum

Adult B. malayi cultivated in the peritoneal cavity of jirds (Meriones unguiculatus) were derived from TRS Laboratories
(Athens, Georgia, USA) and maintained at the Liverpool School of Tropical Medicine. Infected animals received tetracycline at $2.5 \mathrm{mg} / \mathrm{ml}$ in drinking water for a period of 6 weeks. Control infected jirds were maintained in a similar fashion but without the tetracycline. Two weeks after the end of the treatment, worms were collected from the peritoneal cavities using preheated $\left(37^{\circ} \mathrm{C}\right)$ culture medium RPMI-1640 (GIBCO). All procedures involving the use of laboratory animals were approved by the Ethics and Animal Care Committees of the University of Liverpool and Liverpool School of Tropical Medicine, and were carried out according to the Animals (Scientific Procedures) Act (UK Home Office).

Ae. albopictus mosquito cell lines C6/36 (wAlbB) infected with Wolbachia strain wAlbB and non-infected C6/36 (NI) are maintained in culture at the Liverpool School of Tropical Medicine, Liverpool, UK [10]. Cells were cultivated routinely in $25 \mathrm{~cm}^{2}$ plastic culture flasks at $26^{\circ} \mathrm{C}$ in $5 \mathrm{ml}$ of Leibovitz-15 medium containing $2 \mathrm{mM}$ L-glutamine (Life Technologies), $1 \%$ non-essential amino acids, $2 \%$ tryptose phosphate broth, $50 \mathrm{U} / \mathrm{ml}$ penicillin, $50 \mu \mathrm{g} / \mathrm{ml}$ streptomycin (Sigma) and 5\% heat-inactivated FBS (Perbio). Cells were sub-cultured into new flasks every 4-5 days.

Serum samples from $W$. bancrofti infected individuals were collected as part of a clinical trial in Tanzania. The samples used for serology were collected prior to any drug treatment. The study was approved by the ethics research committees of the National Institute for Medical Research, Dar es Salaam, Tanzania, and the Liverpool School of Tropical Medicine, Liverpool, UK. Written and oral informed consent was obtained from all participants [11].

\section{Fractionation of mosquito cells}

Mosquito cells were homogenised in PBS buffer by vortexing with $3 \mathrm{~mm}$ borosilicate glass beads. Homogenates were then centrifuged for $5 \mathrm{~min}$ at $300 \times \mathrm{g}$ to eliminate cell debris. Supernatants containing intracellular components were pelleted by centrifugation at $8,150 \times \mathrm{g}$ for $5 \mathrm{~min}$, and resuspended in $330 \mu \mathrm{l}$ PBS. Cellular suspensions were sonicated on ice for a period of $1 \mathrm{~min}(1 \mathrm{~s} \mathrm{run} / 2 \mathrm{~s}$ pause $)$ at $1,800 \mathrm{~J}$ (cumulative value). The supernatants were pooled and cellular complexes were removed by centrifugation at $8,150 \times \mathrm{g}$ for $10 \mathrm{~min}$. The supernatant was overlaid on $1 \mathrm{ml}$ of sucrose solution and cellular membranes were pelleted from the supernatants by ultracentrifugation in TLA100 (Beckman) at $430,000 \times \mathrm{g}$ for $1 \mathrm{~h}$ at $4^{\circ} \mathrm{C}$. The proteins were precipitated from the supernatant to obtain the cytosolic fraction and from the pellet for the membrane fraction using acetone. Samples were stored at $-80^{\circ} \mathrm{C}$ until use.

\section{Western blot}

Antibodies to wBmPAL were generated as described previously [9]. Anti-VirB6 antibodies were produced by New England Biolabs, (USA) using a similar procedure. 
B. malayi worms were collected from antibiotic-treated and untreated jirds, washed three times in PBS and proteins were extracted using sonication. Fractions of C6/36 cells and intact cell pellets were collected in the separate tubes and proteins were extracted using sonication. The protein concentration was estimated by bicinchoninic acid assay (Invitrogen) following the manufacturer's instructions. Protein extracts of worms were mixed with LDS sample buffer (NuPAGE; Invitrogen), boiled, and run with a 12\% PAGE gel. Protein was transferred to nitrocellulose membranes and used in the Western blot as previously described [12].

\section{Microscopy}

B. malayi adult females were fixed using $4 \%$ formaldehyde in PBS with $0.05 \%$ Triton-X100 (PBST) for $20 \mathrm{~min}$ for confocal microscopy analysis of the localisation of the proteins. During fixation, worms were cut to improve diffusion of the fixative and antibodies. Samples were then washed three times in PBST and treated with RNase A $(100 \mu \mathrm{g} / \mathrm{ml})$ at $36^{\circ} \mathrm{C}$ for $1 \mathrm{~h}$. Then samples were washed in PBS and blocked with 5\% BSA for $15 \mathrm{~min}$ and incubated overnight at $4^{\circ} \mathrm{C}$ with anti-VirB6 antibody diluted 1:200. Secondary antibody labelled with FITC was used at 1:500. After incubation with antibodies samples were co-stained with propidium iodide for 20 min to visualise DNA (host nuclei and Wolbachia) and were viewed with an LSM 5 Pascal confocal microscope (Zeiss).

For immuno-TEM, worms and C6/36 cells were fixed by $4 \%$ paraformaldehyde dissolved in PBS for $4 \mathrm{~h}$ at $4^{\circ} \mathrm{C}$. During fixation, worms were cut. After fixation, samples were washed in PBS (three times on ice). Fixed mosquito cells were embedded into low-melting agarose. All samples were then dehydrated in a series of ethanol concentrations (50-100\%) on ice. Dehydrated samples were embedded in Lowicryl Gold plastic resin. Ultrathin sections were blocked using 5\% BSA and incubated with primary antibodies diluted $1: 100$ in $1 \%$ BSA overnight at $4^{\circ} \mathrm{C}$. The next day, sections were washed three times in PBS and incubated with secondary antibody labelled with $15 \mathrm{~nm}$ gold particles. Sections were washed and contrasted using uranyl acetate (1\%) and lead citrate and analysed under the Tecnai G2 Spirit BioTWIN TEM (the TEM unit, University of Liverpool, UK). For TIVSS analysis serial sections were taken every 70-90 nm to cover the entire bacterial cell of non-dividing Wolbachia.

\section{High resolution mass spectrometric analysis of total parasite protein Proteomics}

For proteomic analysis, a total of 18 adult female B. malayi were collected by washing out the peritoneal cavity of Mongolian jirds (Meriones unguiculatus) with warm RPMI 1640 media not containing FBS. Worms were processed by sonication (in $25 \mathrm{mM}$ ammonium bicarbonate) at $70 \%$ amplitude for $30 \mathrm{~s}$ with $30 \mathrm{~s}$ rest on ice (a total of three cycles). Proteins were treated with the surfactant $0.1 \%(\mathrm{v} / \mathrm{v})$ RapiGestTM (Waters) at $80^{\circ} \mathrm{C}$ for $10 \mathrm{~min}$ followed by reduction with diothiothreitol (DTT) at a final concentration of $3 \mathrm{mM}\left(60^{\circ} \mathrm{C}\right.$ for $\left.10 \mathrm{~min}\right)$ and alkylation with iodoacetamide (IAA) at a final concentration of $50 \mathrm{mM}$ (room temp, in the dark, $1 \mathrm{~h}$ ). The enzyme trypsin (sequencing grade, Promega) was added at an enzyme: substrate ratio of 1:50 and incubated overnight at $37^{\circ} \mathrm{C}$. The surfactant was inactivated the following day by treatment with $0.1 \%$ trifluoroacetic acid (TFA) $\left(37^{\circ} \mathrm{C}\right.$ for $\left.1 \mathrm{~h}\right)$, peptides were recovered following centrifugation at 13,0000 g. A proportion of unfractionated peptide sample was retained for mass spectrometric analysis. A total of $150 \mu \mathrm{g}$ of digested protein material was subjected to extensive fractionation over the $\mathrm{pH}$ range 3-10 using the Agilent 3100 OFFGEL fractionator system as per manufacturer's instructions.

\section{Reversed-phase liquid chromatography- tandem mass spectrometry (RPLC-MSMS)}

Both the unfractionated and fractionated peptide samples were separated by RPLC using a DIONEX UltiMateTM 3000LC chromatography system and MSMS analysis performed on an LTQ Orbitrap Velos using Xcalibur software v2.1 (Thermo Scientific, UK). Peptides $(10 \mu \mathrm{l}=\sim 500 \mathrm{ng}$ ) were injected onto the analytical column (Dionex Acclaim ${ }^{\circ}$ PepMap RSLC C18, $2 \mu \mathrm{m}, 100 \AA$, $75 \mu \mathrm{m}$ i.d. $\times 15 \mathrm{~cm}$, nanoViper.), which was maintained at $35^{\circ} \mathrm{C}$ and at a nanoflow rate of $0.3 \mu \mathrm{lmin}^{-1}$. Peptides were separated over linear chromatographic gradients composed of buffer A $(2.5 \%$ acetonitrile [ACN]: $0.1 \%$ formic acid [FA]) and buffer $\mathrm{B}$ (90\% ACN: 0.1\% FA). Two gradients, 60 (3-50\% buffer B in $40 \mathrm{~min}$ ) and $180 \mathrm{~min}$ (3-60\% buffer B in $140 \mathrm{~min}$ ), were employed for analysis. Full scan MS spectra were acquired over the $\mathrm{m} / \mathrm{z}$ range of $350-2000$ in positive polarity mode by the Orbitrap at a resolution of 30,000. A datadependent Top20 collision induced dissociation (CID) data acquisition method was used. The ion-trap operated with CID MSMS on the 20 most intense ions (above the minimum MS signal threshold of 500 counts).

\section{Protein identification}

All MSMS data generated was searched against a customised database for Wolbachia $(w B m)$ and B. malayi (concatenated from .fasta files obtained from UniProtKB www.uniprot.org, downloaded on 14/01/2013) using the search engine MASCOT and Proteome Discoverer software v1.2 (Thermo Scientific, UK). Search parameters included a precursor mass tolerance of $10 \mathrm{ppm}$ and fragment ion tolerance $0.8 \mathrm{Da}$ with one tryptic missed cleavage permitted. Carbamidomethyl (C) was set as a static modification with oxidation of methionine $(\mathrm{M})$, deamidation (N, Q) and phospho (ST) (Y) set as dynamic 
modifications. A decoy database was searched and relaxed peptide confidence filters applied to the dataset (ion scores $p<0.05 /$ FDR $5 \%$ ).

\section{IgG antibody ELISA}

Flat-bottomed 96-well ELISA plates (Immulon ${ }^{\circ} 4 \mathrm{HBX}$ ultra high binding polystyrene microtiter plates) were coated overnight at $4^{\circ} \mathrm{C}$ with $50 \mu \mathrm{l} /$ well of $5 \mu \mathrm{g} / \mathrm{ml}$ of Wolbachia (PAL and VirB6) recombinant proteins diluted in carbonate-bicarbonate buffer, $\mathrm{pH}$ 9.6. Plates were covered with plastic film to prevent drying. The following day, plates were blocked with $5 \%$ skimmed milk powder in PBST (0.05\% Tween 20 in PBS, pH 7.4), covered as previously and incubated for $1 \mathrm{~h}$ at $37^{\circ} \mathrm{C}$. Plates were then inverted and washed with PBST. Human serum samples $(50 \mu \mathrm{l} /$ well $)$, at a final dilution of 1:200 in blocking buffer, were added to the plates, which were then covered and incubated for $2 \mathrm{~h}$ at room temperature (RT). Plates were inverted and washed. $50 \mu \mathrm{l} /$ well of primary antibody murine anti-human IgG (1 $\mathrm{mg} / \mathrm{ml}$; Skybio, UK) diluted 1:1000 in PBST were added to separate plates, which were then covered and incubated for $1 \mathrm{~h}$ at RT. After inversion and washing, $75 \mu \mathrm{l} /$ well of secondary antibody anti-murine rabbit IgG conjugated with horseradish peroxidase (HRP) at $1 \mathrm{mg} / \mathrm{ml}$ (Invitrogen) diluted 1:2000 in PBT was added to each plate. Plates were covered and incubated for $1 \mathrm{~h}$ at RT. After inversion and washing, $100 \mu \mathrm{l} /$ well of TMB substrate and chromogen solution (Sigma) was added to each plate and these were incubated at RT in the dark until the reaction was stopped after $30 \mathrm{~min}$ by adding $50 \mu \mathrm{l} /$ well of $1 \mathrm{M}$ $\mathrm{H}_{2} \mathrm{SO}_{4}$. The values of optical density were read at $450 \mathrm{~nm}$ wavelength. Cut-off values were then defined as three times the standard deviation plus the mean of the European control sera for each antigen tested. The Mann Whitney test (used to compare the differences in median antibody levels between the different clinical groups) was carried out using GraphPad Prism (5.0a Mac OS X).

\section{Results}

Identification of Wolbachia lipoproteins in Brugia malayi adult female worms by proteomic and mass spectrometric analysis Using a global proteomics approach coupled with high resolution mass spectrometric analysis of protein extract, we were able to confirm the presence of two predicted Wolbachia lipoproteins in adult female B. malayi. We have detected outer membrane protein, PAL (Wbm0152) and TIVSS, VirB6 components (Wbm0794) with high confidence, following manual validation of MSMS data. Wolbachia proteins have been ranked according to protein sequence coverage observed and the top 20 proteins from this ranked list are shown in Figure 1. The highest degree of lipoprotein sequence coverage was observed for PAL ( 20\%) with VirB6 (Wbm0794) confirmation based upon single peptide identification, with reduced $(<1 \%)$ protein sequence coverage. We were also able to successfully differentiate between two paralogues of the TIVSS components, VirB6 (Wbm0793 and Wbm0794) by unique peptide identification. Both paralogues were identified with similar reduced $(<1 \%)$ protein sequence coverage. Paralogue Wbm0793 was not predicted as a lipoprotein by any of the bioinformatic tools used in this study (Database of Lipoproteins, LIPO, LipoP).

\section{Localisation of Wolbachia Peptidoglycan-Associated Lipoprotein (wPAL) in Aedes albopictus mosquito cells and Brugia malayi adult worms}

We used an affinity purified polyclonal rabbit antiwBmPAL antibody to perform western blot analysis of the proteins extracted from the Wolbachia-infected mosquito cell line C6/36 (wAlbB) and B. malayi ( $w \mathrm{Bm})$ females. As demonstrated previously, protein extracts from B. malayi females showed a single band at $17 \mathrm{kDa}$, which was lost following depletion of Wolbachia with tetracycline (Figure 2A, [9]). Aedes albopictus mosquito cells (C6/36 line) were crushed to extract total proteins, cytosolic protein fractions, and membrane protein fractions for western blot analysis (Figure 2B). The crude extract of the cells infected with $w \mathrm{AlbB}$ and the membrane fraction from $\mathrm{C} 6 / 36$ ( $w \mathrm{AlbB}$ ) showed positive signals for PAL, whereas the cytosolic fraction from $\mathrm{C} 6 / 36$ ( $w \mathrm{AlbB})$ showed no reactivity. A slight difference in migration of PAL in lanes 3 and 4 may relate to differences in preparation of the extracts with enriched membrane proteins running slightly slower in the gel. Non-infected C6/36 (NI) cells were used as a negative control and the protein extracts from all fractions showed no wPAL reactivity (Figure $2 \mathrm{~B}$ ).

Next we investigated the ultrastructural localisation of wBmPAL in B. malayi females using transmission electron microscopy. We observed a specific localisation of antiwBmPAL antibodies to the membranes of Wolbachia (Figure 2C, D). Immunogold particles were not observed on the membranes of vacuoles containing Wolbachia. Statistical analysis of the distribution of immunogold particles on the sections of B. malayi lateral cord showed $86 \%$ of all counted particles located on the bacterial membrane and only $14 \%$ in the cytoplasm of the host cell (Table 1). The low number of immunogold particles that were randomly distributed in the cytoplasm represented the level of background staining and this level was similar in all the control experiments (tetracycline treated B. malayi and Wolbachia-free Acanthocheilonema viteae, Table 1).

Localisation of Wolbachia VirB6, a core component of Type IV Secretion System in Aedes albopictus mosquito cells and Brugia malayi adult worms

Antibodies raised to $\mathrm{wBmVirB6}$, were unsuitable for western blot analysis due to non-specific binding. Immunogold 


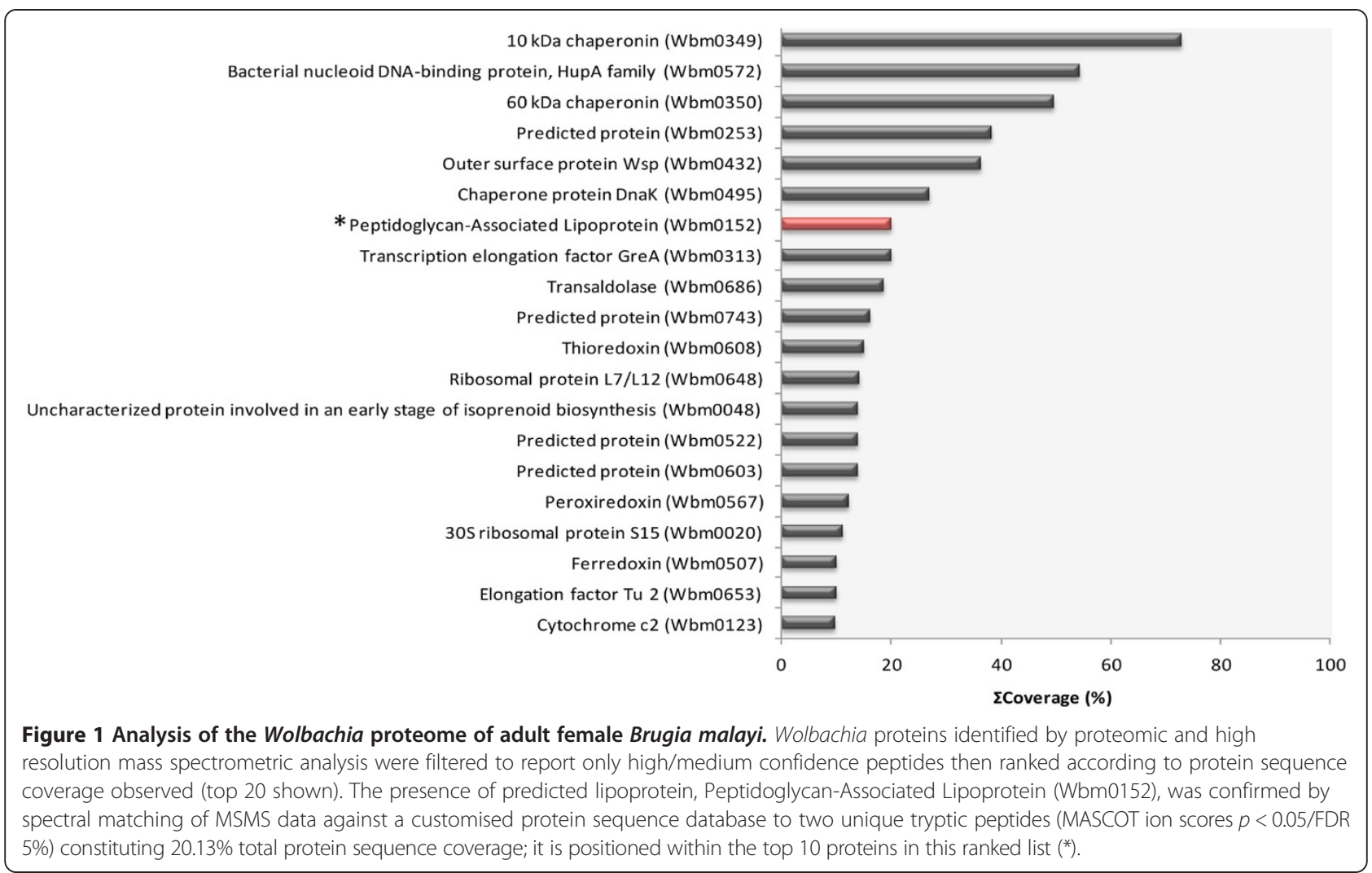

ultrastructural localisation of VirB6 in host cells was determined using immuno-TEM analysis of $B$. malayi females and C6/36 (wAlbB) cells. Wolbachia VirB6 protein localised as a single discreet cluster(s) on the Wolbachia membranes (Figure 3A, B). In addition, occasional immunogold labelling was also observed localised within the bacterial matrix (Figure 3C, D), which could represent a precursor of the protein synthesised in bacteria. This pattern of protein distribution was similar in somatic cells and developing embryos of B. malayi (Figure 3A-D). Serial sections of bacteria determined that the VirB6 protein clusters as a single complex established in bacterial membranes and on some sections near the pole of bacteria. In some cases Wolbachia had two clusters, which were located on the opposite poles prior to division.

\section{Confocal microscopy}

To determine whether the protein localisation was associated with Wolbachia location in whole organisms or cells, we used whole mount confocal microscopy. The location of antibodies to both wBmPAL and wBmVirB6 were associated with Wolbachia (stained by propidium iodide, Figure 4A-F) in the hypodermal cord cells of B. malayi. Antibody reactivity was absent from tetracycline treated B. malayi (Figure 4G-I).

\section{Serology}

Next, we investigated the human IgG response to the two lipoproteins in people infected with $W$. bancrofti from Tanzania. Both Wolbachia lipoproteins showed high levels and frequency of antibody reactivity in infected individuals (Figure 5) with $98.4 \%$ and $82.3 \%$ of patients demonstrating IgG responses to wBmPAL and wBmVirB6 respectively.

\section{Discussion}

Here we show that two Wolbachia lipoproteins, PAL and VirB6 are located on the membranes of Wolbachia ( $w \mathrm{Bm}$ and $w \mathrm{AlbB}$ ). Proteomic analysis of B. malayi adult female protein extracts confirmed the presence of two lipoproteins, previously predicted through bioinformatics; Wolbachia peptidoglycan associated lipoprotein (wBmPAL) and the Type IV Secretion System component, VirB6 (wBmVirB6). Using protein sequence coverage as an estimate of relative abundance, we showed that wBmPAL was among the most abundant Wolbachia proteins present in an extract of adult female worms with wBmVirB6 only detected at a much lower abundance. This high abundance of PAL has been previously reported in adults and microfilariae of $B$. malayi [13] and $O$. ochengi $[14]$ in which PAL consistently appears in the top 10 abundant Wolbachia proteins. This differential 




abundance was reflected in the immunogold-labelling, which showed wBmPAL localised at numerous sites within the bacterial membranes, whereas wBmVirB6 was present as a single cluster on each bacterial cell and also located within the bacterial membranes. Immunoblotting of fractionated extracts confirmed the localisation of PAL to membranes and its absence from cytosolic fractions of C6/36 mosquito cells infected with the $w$ AlbB strain.
Our observations are consistent with previous studies, which localise PAL to the membranes of other gramnegative bacteria such as E. coli [15]. The typical cell envelope structure of gram-negative bacteria consists of three layers: outer and inner membranes with a thin layer of peptidoglycan (PG) located between the membranes (the periplasmic space). The N-terminus of PAL containing the lipid moiety is anchored to the inner face of the outer membrane, with the C-terminus binding to 
Table 1 Statistical analysis of gold-dot distribution on the TEM sections of $B$. malayi and $A$. viteae

\begin{tabular}{lll}
\hline \multicolumn{3}{l}{ Immunogold distribution on the sections stained with anti-PAL antibody } \\
\hline BM & $86.6 \pm 18.5^{*}, \wedge, "$ & Host cytoplasm (mean \pm SD, \%) \\
BM-TET 0 & $13.4 \pm 11.7$ \\
AV & 0 & $11.7 \pm 5.0$ \\
\end{tabular}

* - Statistical analysis of means of immunogold particles located on bacterial material and particles located on host cytoplasm in B. malayi (BM), p-value $<0.01$. $\wedge$ - Statistical analysis of means of immunogold particles located on bacterial material in B. malayi (BM) and particles located on host cytoplasm of tetracycline treated Brugia malayi (BM-TET) p-value $<0.01$.

" - Statistical analysis of means of immunogold particles located on bacterial material in B. malayi (BM) and particles located on host cytoplasm of $A$. viteae (AV) p-value $<0.01$

PG via a pocket for m-DAP residues crosslinking the outer membrane to PG [16]. However, Wolbachia does not contain the entire biosynthesis pathway for PG [17], and electron microscopy analysis of the Wolbachia cell wall confirms the absence of a PG layer $[18,19]$. Some components of the biosynthesis of PG are still intact, for example, lipid II, which, in addition to its role in the cell envelope architecture, has a role in cell division [11]. In gram-negative bacteria PAL anchors to the outer membrane but is located in the periplasmic space as part of the Tol-PAL complex $[20,21]$ and evidence for the existence of the periplasmic component of the Tol transport systems (Wbm5057) is found in $w \mathrm{Bm}$. This system plays important roles in the transport of molecules through the outer membrane of bacteria, in the regulation of the expansion of membranes and in bacterial sensitivity to antibiotics [21]. The complex is also involved in the protection of bacteria against the penetration of phage particles into the cell and in the formation of cell envelopes in daughter cells $[22,23]$.

The lipoprotein, VirB6, was also localised to the membranes of Wolbachia in both wAlbB and $w \mathrm{Bm}$. In contrast to PAL, which was widely distributed throughout the membranes, VirB6 was localised as a discreet single complex on the bacterial cell. Through the use of serial
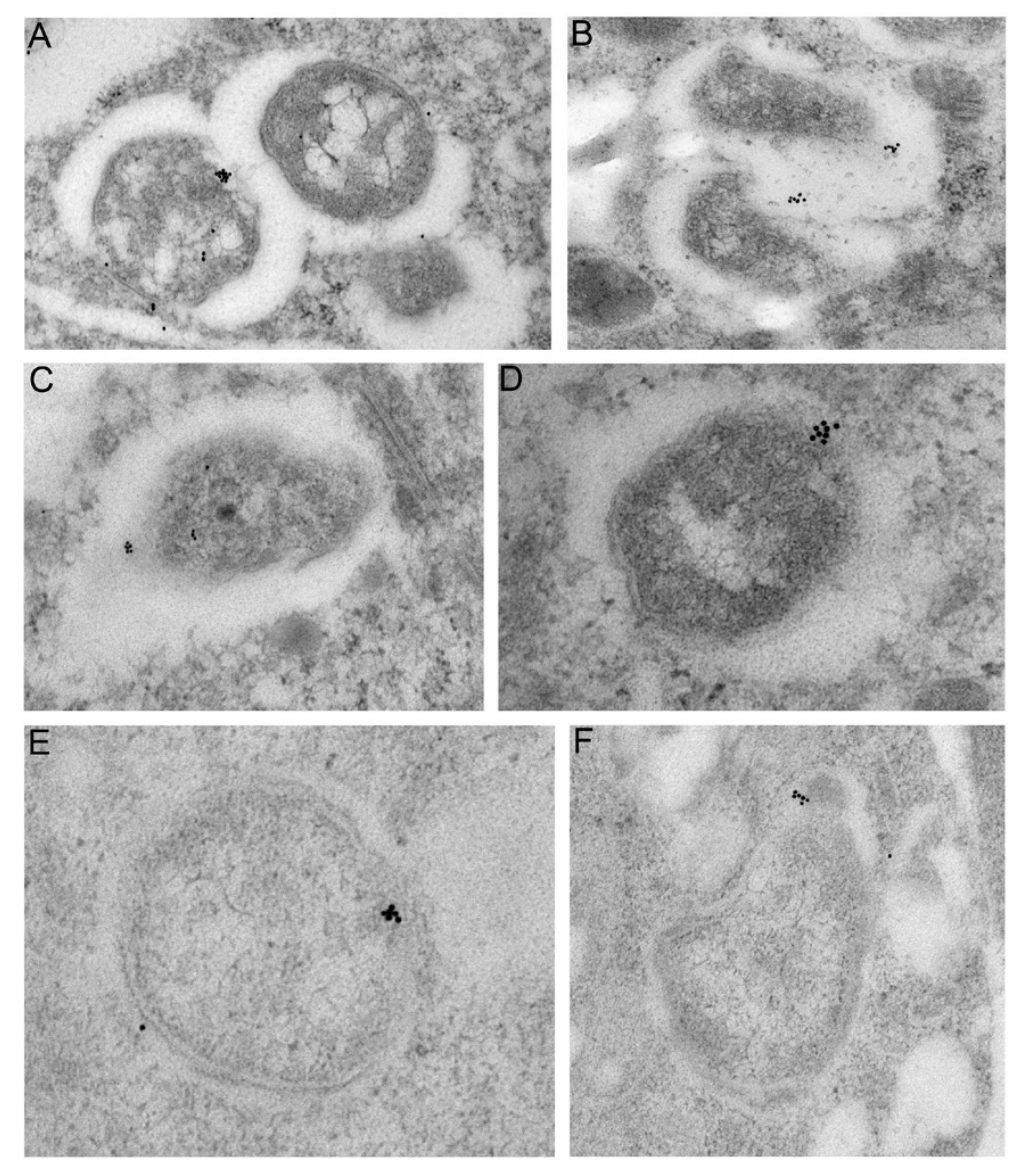

Figure 3 Ultrastructural distribution of Wolbachia VirB6 in lateral cord of Brugia malayi. Immuno-transmission electron microphotographs showing distribution of Wolbachia VirB6 in lateral cord of Brugia malayi and a mosquito cell line. A, B - localisation of VirB6 in a bacterium-bacterium connection in cytoplasm of lateral cord of adult B. malayi, C, D - a single complex of VirB6 detected on the bacterial pole in developing microfilaria, E, F - VirB6 on wAlbB bacterial membrane in cytoplasm of mosquito cells (C6/36 cell line). 

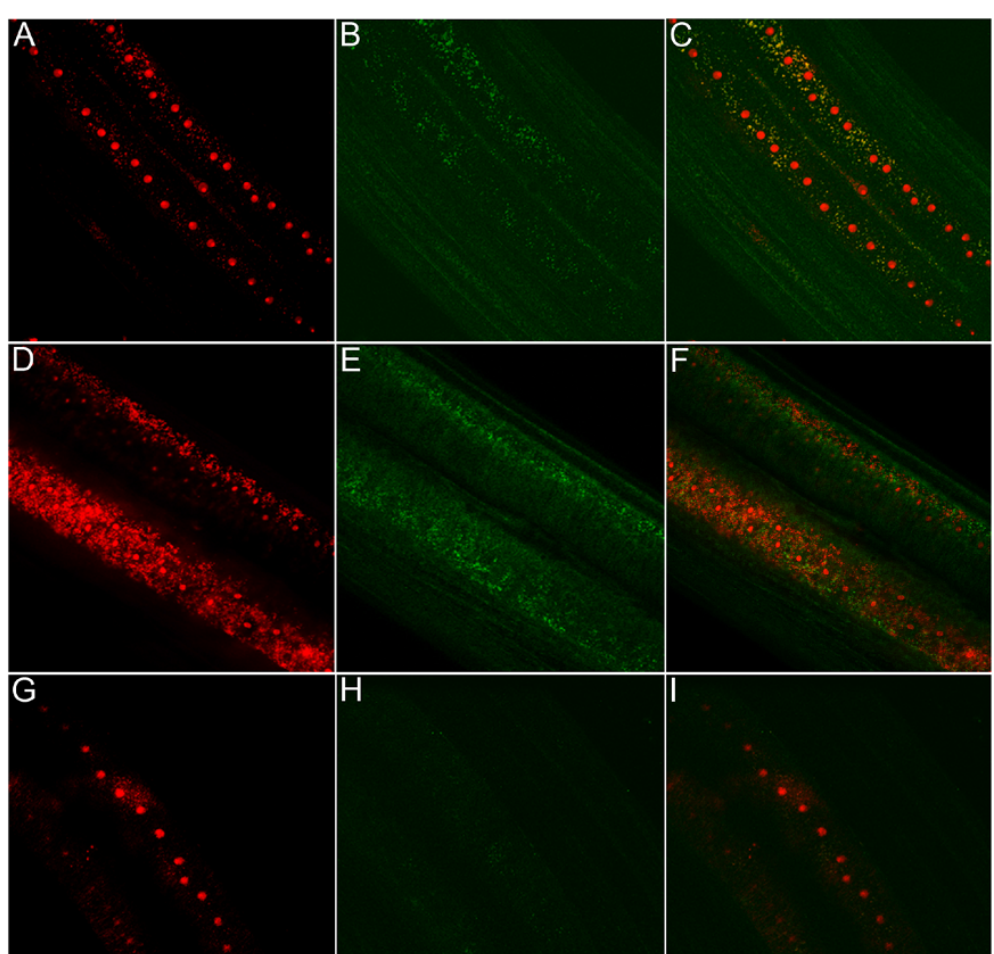

Figure 4 Visualisation of PAL and VirB6 in whole worm mounts of B. malayi. A, B, Wolbachia (small red foci, bacteria DNA stained by propidium iodide, large red spot, nematode nuclei, see Methods) and PAL (green, secondary antibody labeled by FITC) in lateral cord of filarial nematode, C, merged A and B. D, E, VirB6 (green) and Wolbachia (small red foci) in B. malayi (wBm). F, merged D and E. G, H, negative control - Wolbachia-free B. malayi treated with antibiotic (lack of PAL reactivity shown as representation of both antibodies). I, merged $\mathbf{G}$ and $\mathbf{H}$

sectioning at intervals of 70-90 $\mathrm{nm}$ and covering the entire bacterial cell of non-dividing Wolbachia, we observed that Wolbachia has a single TIVSS complex for each cell. This is also observed for the TIVSS complex in Agrobacterium [24-26]. During bacterial division, the components of the secretion system migrate onto the pole of Agrobacterium [27,28]. On the micrographs of the sections for both insect cells and filarial nematodes, VirB6 was found on the membranes of the Wolbachia forming a single complex close to the pole of the bacterium. In detailed analysis of immuno-TEM sections, the complex of VirB6 proteins on the bacterial membrane were often observed in close association with the vacuolar membrane.

The bacterial VirB6 protein is a core component of TIVSS and plays important roles in the transfer of DNA-protein complexes to induce bacterial adaptation processes, to deliver pathogens into host cytoplasm, and to establish bacterial effects on host biology [28-31]. In gram-negative bacteria, TIVSS connects two bacterial membranes and forms a canal-like structure [30]. Previous analysis of the Wolbachia VirB6 gene in genomes of
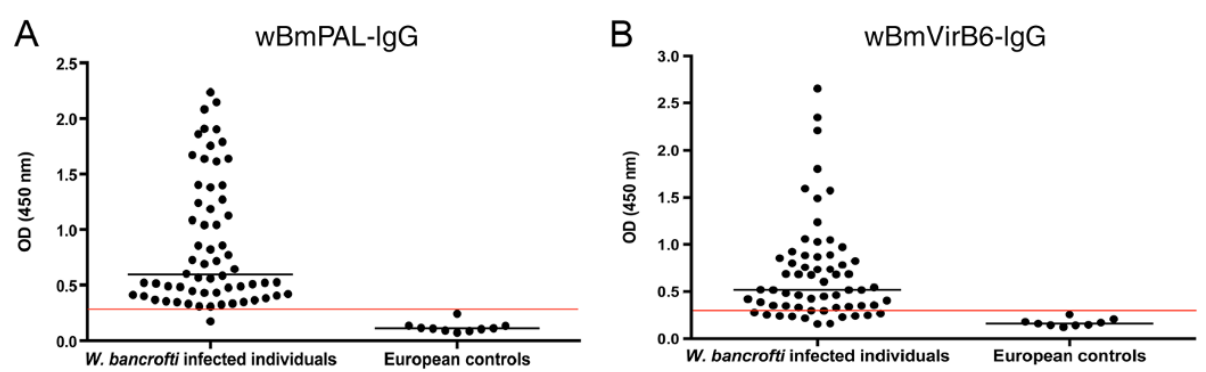

Figure 5 lgG antibody responses against Wolbachia lipoproteins in patients infected with Wuchereria bancrofti. IgG reactivity against WBmPAL (A) and virB6 (B). Infected groups both show significantly elevated levels of IgG to both lipoproteins $(P<0.05)$ compared with European controls. The red line represents the cutoff value (mean +3 X SD). 
different Wolbachia strains showed several predicted isoforms [32]. In this study we identified two isoforms from proteomic analysis, only one of which (Wbm0794) was predicted as a lipoprotein by bioinformatics. Computational analysis of the structure of VirB6 protein revealed that the 5-7 transmembrane domains were located on the inner bacterial membrane [30]. The TIVSS is likely to function as a major pathway for the secretion of bacterial products, which are involved in the Wolbachia-host symbiosis and a number of Wolbachia proteins, including both lipoproteins, can be detected in the parasite secretome [33]. Rickettsial bacteria related to Wolbachia such as Anaplasma and Ehrlichia use TIVSS for successful infection of mammalian cells [29].

The lipoprotein biosynthesis pathway of Wolbachia has been investigated as a potential chemotherapeutic target for filariasis [34]. Treatment of Wolbachia-infected mosquito cells with the signal peptidase II (LspA) inhibitor, globomycin, led to a dose-dependent reduction in Wolbachia load preceded by an inhibition of lipoprotein processing. Treatment of B. malayi with globomycin in vitro resulted in significant reductions in motility and viability validating this as a target for drug discovery. Similarly, treatment of the related bacteria Ehrlichia chaffeensis with globomycin, prevented infection of HL-60 in vitro, which was associated with inhibition of lipoprotein processing [35]. PAL specifically has been implicated in this infection process, as antibodies to PAL (OmpA) bind to E. chaffeensis and prevent infection of cells in vitro [36]. Furthermore PAL of Helicobacter pylori, known as OprL, has been defined as a critical drug target in silico [37] suggesting that targeting PAL and/or lipoprotein biosynthesis could deliver broad spectrum antibiotic activity.

Wolbachia lipoproteins are the agonists of inflammatory pathogenesis of filarial disease through activation of innate and adaptive immunity via recognition by TLR $2 / 6$ $[6,7]$. Bacterial lipoproteins play an important role in the pathogenesis of several bacterial infections including Yersinia pestis, Pseudomonas aeruginosa, Salmonella enterica, Nesseiria meningitidis, Borrelia burdorferi and Mycobacterium tuberculosis [38]. In sepsis, E. coli PAL is a potent TLR2 agonist, which contributes to inflammation, cardiac dysfunction, endothelial activation, coagulopathy, and vascular leakage [39-42]. In filariasis Wolbachia PAL recruits neutrophils, macrophages and other innate immune cells, which accumulate around living Onchocerca adults worms in onchocermata $[43,44]$ and in response to dying microfilariae in the cornea $[45,46]$, which stimulates their activation and the production of an array of pro-inflammatory cytokines and mediators $[6,9]$. Wolbachia lipoproteins also drive adaptive Th1 immunity through activation of dendritic cells [9]. In view of the relative abundance of PAL compared to VirB6, it seems likely that PAL is the major driver of Wolbachia-mediated inflammatory immunity. Exposure of the host to lipoproteins will occur on the release of Wolbachia on the death of the parasite or through their presence in the parasite secretome [33]. The high level and frequency of antibodies against these proteins in patients infected with $W$. bancrofti is supportive of their inherent immunogenicity and exposure to the adaptive immune system.

\section{Conclusion}

Two Wolbachia lipoproteins, wBmPAL and wBmVirB6, are present in extracts of Brugia malayi with wBmPAL among the most abundant of Wolbachia proteins. Both lipoproteins localised to bacterial membranes with wBmVirB6 present as a single cluster suggesting a single Type IV Secretory System on each Wolbachia cell. Serological analysis showed that both proteins were immunogenic and raised antibody responses in the majority of individuals infected with Wuchereria bancrofti.

\section{Competing interests}

The authors declare that they have no competing interests.

\section{Authors' contributions}

DV designed the study and carried out microscopy and western blotting and wrote the first draft of the manuscript, AG performed ELISA analysis, GRM performed proteomic analysis, KJ participated in the design of the study and data analysis, LF participated in the design of the study and assisted with data analysis and interpretation, MJT conceived and designed the study and wrote the final draft of the manuscript. All authors have read and approved the final manuscript.

\section{Acknowledgements}

This work was supported by a Bill and Melinda Gates Foundation (BMGF) funded $A \bullet W O L$ drug development programme. We thank Barton Slatko and Bo Wu (NEB) for provision of VirB6 protein and antibody.

Received: 1 August 2014 Accepted: 27 September 2014

Published online: 06 October 2014

References

1. Taylor MJ, Bandi C, Hoerauf A: Wolbachia bacterial endosymbionts of filarial nematodes. Adv Parasitol 2005, 60:245-284.

2. Zug R, Hammerstein P: Still a host of hosts for Wolbachia: analysis of recent data suggests that $40 \%$ of terrestrial arthropod species are infected. PLoS One 2012, 7:e38544.

3. Landmann F, Voronin D, Sullivan W, Taylor MJ: Anti-filarial activity of antibiotic therapy is due to extensive apoptosis after Wolbachia depletion from filarial nematodes. PLoS Pathog 2011, 7:e1002351.

4. Slatko BE, Taylor MJ, Foster JM: The Wolbachia endosymbiont as an anti-filarial nematode target. Symbiosis 2010, 51:55-65.

5. Taylor MJ, Hoerauf A, Townson S, Slatko BE, Ward SA: Anti-Wolbachia drug discovery and development: safe macrofilaricides for onchocerciasis and lymphatic filariasis. Parasitology 2014, 141(1):119-127.

6. Tamarozzi F, Halliday A, Gentil K, Hoerauf A, Pearlman E, Taylor MJ: Onchocerciasis: the role of Wolbachia bacterial endosymbionts in parasite biology, disease pathogenesis, and treatment. Clin Microbiol Rev 2011, 24(3):459-468.

7. Turner JD, Langley RS, Johnston KL, Gentil K, Ford L, Wu B, Graham M, Sharpley F, Slatko B, Pearlman E, Taylor MJ: Wolbachia lipoprotein stimulates innate and adaptive immunity through Toll-like receptors 2 and 6 to induce disease manifestations of filariasis. J Biol Chem 2009, 284:22364-22378. 
8. Hise AG, Daehnel K, Gillette-Ferguson I, Cho E, McGarry HF, Taylor MJ, Golenbock DT, Fitzgerald KA, Kazura JW, Pearlman E: Innate immune responses to endosymbiotic Wolbachia bacteria in Brugia malayi and Onchocerca volvulus are dependent on TLR2, TLR6, MyD88, and Mal, but not TLR4, TRIF, or TRAM. J Immunol 2007, 178:1068-1076.

9. Rances E, Voronin D, Tran-Van V, Mavingui P: Genetic and functional characterization of the type IV secretion system in Wolbachia. J Bacterio 2008, 190:5020-5030.

10. Turner JD, Langley RS, Johnston KL, Egerton G, Wanji S, Taylor MJ: Wolbachia endosymbiotic bacteria of Brugia malayi mediate macrophage tolerance to TLR- and CD40-specific stimuli in a MyD88/ TLR2-dependent manner. J Immunol 2006, 177:1240-1249.

11. Taylor MJ, Makunde WH, McGarry HF, Turner JD, Mand S, Hoerauf A: Macrofilaricidal activity after doxycycline treatment of Wuchereria bancrofti: a double-blind, randomised placebo-controlled trial. Lancet 2005, 365(9477):2116-2121.

12. Voronin D, Cook DA, Steven A, Taylor MJ: Autophagy regulates Wolbachia populations across diverse symbiotic associations. Proc Natl Acad Sci U S A 2012, 109:E1638-E1646.

13. Bennuru S, Meng Z, Ribeiro JM, Semnani RT, Ghedin E, Chan K, Lucas DA, Veenstra TD, Nutman TB: Stage-specific proteomic expression patterns of the human filarial parasite Brugia malayi and its endosymbiont Wolbachia. Proc Natl Acad Sci U S A 2011, 108(23):9649-9654.

14. Darby AC, Armstrong SD, Bah GS, Kaur G, Hughes MA, Kay SM, Koldkjær P, Rainbow L, Radford AD, Blaxter ML, Tanya VN, Trees AJ, Cordaux R, Wastling $J M$, Makepeace BL: Analysis of gene expression from the Wolbachia genome of a filarial nematode supports both metabolic and defensive roles within the symbiosis. Genome Res 2012, 22(12):2467-2477.

15. Cascales E, Bernadac A, Gavioli M, Lazzaroni JC, Lloubes R: Pal lipoprotein of Escherichia coli plays a major role in outer membrane integrity. J Bacteriol 2002, 184:754-759.

16. Bos MP, Robert $\mathrm{V}$, Tommassen J: Biogenesis of the gram-negative bacterial outer membrane. Ann Rev Microbio/ 2007, 61:191-214.

17. Henrichfreise B, Schiefer A, Schneider T, Nzukou E, Poellinger C, Hoffmann TJ, Johnston KL, Moelleken K, Wiedemann I, Pfarr K, Hoerauf A, Sahl HG: Functional conservation of the lipid II biosynthesis pathway in the cell wall-less bacteria Chlamydia and Wolbachia: why is lipid II needed? Mol Microbiol 2009, 73:913-923.

18. Kozek WJ: Transovarially-transmitted intracellular microorganisms in adult and larval stages of Brugia malayi. J Parasitol 1977, 63:992-1000.

19. Kozek WJ, Marroquin HF: Intracytoplasmic bacteria in Onchocerca volvulus. Am J Trop Med Hyg 1977, 26:663-678.

20. Godlewska R, Wisniewska K, Pietras Z, Jagusztyn-Krynicka EK: Peptidoglycanassociated lipoprotein (Pal) of Gram-negative bacteria: function, structure, role in pathogenesis and potential application in immunoprophylaxis. FEMS Microbiol Lett 2009, 298:1-11.

21. Cascales E, Lloubes R: Deletion analyses of the peptidoglycan-associated lipoprotein Pal reveals three independent binding sequences including a TolA box. Mol Microbiol 2004, 51:873-885.

22. Heilpern AJ, Waldor MK: CTXphi infection of Vibrio cholerae requires the tolQRA gene products. J Bacterio/ 2000, 182:1739-1747.

23. Riechmann L, Holliger P: The C-terminal domain of TolA is the coreceptor for filamentous phage infection of E. coli. Cell 1997, 90:351-360.

24. Jakubowski SJ, Cascales E, Krishnamoorthy V, Christie PJ: Agrobacterium tumefaciens VirB9, an outer-membrane-associated component of a type IV secretion system, regulates substrate selection and T-pilus biogenesis. J Bacteriol 2005, 187:3486-3495

25. Christie PJ, Atmakuri K, Krishnamoorthy V, Jakubowski S, Cascales E: Biogenesis, architecture, and function of bacterial type IV secretion systems. Ann Rev Microbiol 2005, 59:451-485.

26. Christie PJ, Cascales E: Structural and dynamic properties of bacterial type IV secretion systems (review). Mol Membr Biol 2005, 22:51-61.

27. Atmakuri K, Ding Z, Christie PJ: VirE2, a type IV secretion substrate, interacts with the VirD4 transfer protein at cell poles of Agrobacterium tumefaciens. Mol Microbiol 2003, 49:1699-1713.

28. Kumar RB, Das A: Polar location and functional domains of the Agrobacterium tumefaciens DNA transfer protein VirD4. Mol Microbiol 2002, 43:1523-1532

29. Rikihisa Y: Molecular events involved in cellular invasion by Ehrlichia chaffeensis and Anaplasma phagocytophilum. Vet Parasitol 2010, 167:155-166.
30. Fronzes R, Christie PJ, Waksman G: The structural biology of type IV secretion systems. Nat Rev Microbiol 2009, 7:703-714.

31. Durand E, Verger D, Rego AT, Chandran V, Meng G, Fronzes R, Waksman G: Structural biology of bacterial secretion systems in gram-negative pathogens-potential for new drug targets. Infect Disord Drug Targets 2009, 9:518-547.

32. Pichon S, Bouchon D, Cordaux R, Chen L, Garrett RA, Greve P: Conservation of the Type IV secretion system throughout Wolbachia evolution. Biochem Biophys Res Commun 2009, 385:557-562.

33. Bennuru S, Semnani R, Meng Z, Ribeiro JM, Veenstra TD, Nutman TB: Brugia malayi excreted/secreted proteins at the host/parasite interface: stage- and gender-specific proteomic profiling. PLOS NTD 2009, 3:e410.

34. Johnston KL, Wu B, Guimaraes A, Ford L, Slatko BE, Taylor MJ: Lipoprotein biosynthesis as a target for anti-Wolbachia treatment of filarial nematodes. Parasit Vectors 2010, 3:99.

35. Huang $H$, Lin M, Wang $X$, Kikuchi T, Mottaz $H$, Norbeck A, Rikihisa Y: Proteomic analysis of and immune responses to Ehrlichia chaffeensis lipoproteins. Infect Immun 2008, 76:3405-3414.

36. Cheng Z, Miura K, Popov VL, Kumagai Y, Rikihisa Y: Insights into the CtrA regulon in development of stress resistance in obligatory intracellular pathogen Ehrlichia chaffeensis. Mol Microbiol 2011, 82:1217-1234.

37. Neelapu NR, Pavani T: Identification of novel drug targets in HpB38, HpP12, HpG27, Hpshi470, HpSJM180 strains of Helicobacter pylori: an in silico approach for therapeutic intervention. Curr Drug Targets 2013, 14:601-611.

38. Kovacs-Simon A, Titball RW, Michell SL: Lipoproteins of bacterial pathogens. Infect Immun 2011, 79:548-561.

39. Hellman J, Roberts JD Jr, Tehan MM, Allaire JE, Warren HS: Bacterial peptidoglycan-associated lipoprotein is released into the bloodstream in gram-negative sepsis and causes inflammation and death in mice. J Biol Chem 2002, 277:14274-14280.

40. Liang MD, Bagchi A, Warren HS, Tehan MM, Trigilio JA, Beasley-Topliffe LK, Tesini BL, Lazzaroni JC, Fenton MJ, Hellman J: Bacterial peptidoglycanassociated lipoprotein: a naturally occurring toll-like receptor 2 agonist that is shed into serum and has synergy with lipopolysaccharide. J Infect Dis 2005, 191(6):939-948.

41. Zhu X, Bagchi A, Zhao H, Kirschning CJ, Hajjar RJ, Chao W, Hellman J, Schmidt U: Toll-like receptor 2 activation by bacterial peptidoglycanassociated lipoprotein activates cardiomyocyte inflammation and contractile dysfunction. Crit Care Med 2007, 35:886-892.

42. Shin HS, Xu F, Bagchi A, Herrup E, Prakash A, Valentine C, Kulkarni H, Wilhelmsen K, Warren S, Hellman J: Bacterial lipoprotein TLR2 agonists broadly modulate endothelial function and coagulation pathways in vitro and in vivo. J Immunol 2011, 186(2):1119-1130.

43. Brattig NW, Buttner DW, Hoerauf A: Neutrophil accumulation around Onchocerca worms and chemotaxis of neutrophils are dependent on Wolbachia endobacteria. Microbes Infect 2001, 3:439-446.

44. Tamarozzi F, Wright HL: Johnston KL, Edwards SW, Turner JD, Taylor MJ: Human filarial Wolbachia lipopeptide directly activates human neutrophils in vitro. Parasit Immunol 2014, 36(10):494-502.

45. Gillette-Ferguson I, Daehnel K, Hise AG, Sun Y, Carlson E, Diaconu E, McGarry HF, Taylor MJ, Pearlman E: Toll-like receptor 2 regulates CXC chemokine production and neutrophil recruitment to the cornea in Onchocerca volvulus/Wolbachia-induced keratitis. Infect Immun 2007, 75:5908-5915.

46. Gillette-Ferguson I, Hise AG, McGarry HF, Turner J, Esposito A, Sun Y, Diaconu E, Taylor MJ, Pearlman E: Wolbachia-induced neutrophil activation in a mouse model of ocular onchocerciasis (river blindness). Infect Immun 2004, 72:5687-5692.

doi:10.1186/s13071-014-0462-1

Cite this article as: Voronin et al:: Wolbachia lipoproteins: abundance, localisation and serology of Wolbachia peptidoglycan associated lipoprotein and the Type IV Secretion System component, VirB6 from Brugia malayi and Aedes albopictus. Parasites \& Vectors 2014 7:462. 